JOURNAL OF SYNCHROTRON RADIATION

ISSN 1600-5775

Received 4 April 2019

Accepted 21 May 2019

Edited by P. A. Pianetta, Stanford Synchrotron Radiation Laboratory, USA

Keywords: photon-counting; hybrid pixel detectors; detector dead-time; photon pile-up; high photon flux; linearization.

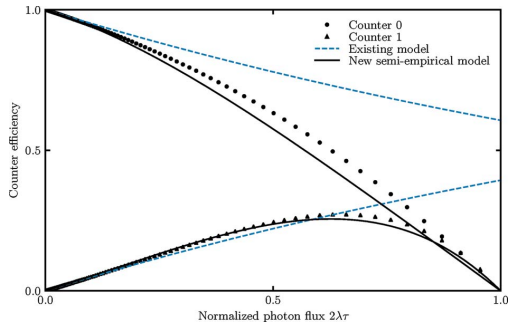

C 2019 International Union of Crystallography

\section{Dead-time correction for spectroscopic photon-counting pixel detectors}

\author{
Gabriel Blaj* \\ SLAC National Accelerator Laboratory, 2575 Sand Hill Road, Menlo Park, CA 94025, USA. \\ *Correspondence e-mail: blaj@slac.stanford.edu
}

Modern photon-counting pixel detectors have enabled a revolution in applications at synchrotron light sources and beyond in the last decade. One of the limitations of the current detectors is their reduced counting linearity or even paralysis at high counting rates, due to dead-time which results in photon pile-up. Existing dead-time and pile-up models fail to reproduce the complexity of dead-time effects on photon-counting, resulting in empirical calibrations for particular detectors at best, imprecise linearization methods, or no linearization. This problem will increase in the future as many synchrotron light sources plan significant brilliance upgrades and free-electron lasers plan moving to a quasicontinuous operation mode. Presented here are the first models that use the actual behavior of the analog pre-amplifiers in spectroscopic photon-counting pixel detectors with constant current discharge (e.g. the Medipix and CPix families of detectors) to deduce more accurate analytical models and optimal linearization methods. In particular, for detectors with at least two counters per pixel, the need for calibration, or previous knowledge of the detector and beam parameters (dead-time, integration time, large sets of synchrotron filling patterns), is completely eliminated. This is summarized in several models of increasing complexity and accuracy. Finally, a general empirical approach is presented, applicable to any particular cases where the analytical approach is not sufficiently precise.

\section{Introduction}

Modern photon science was made possible by the advent of hybrid pixel detectors (Heijne et al., 1988; Anghinolfi et al., 1992), leveraging developments in commercial applicationspecific integrated circuit (ASIC) design while separating it from sensor design. This enabled almost two decades ago the first highly segmented photon-counting pixel detectors, with $64 \mathrm{~K}$ photon-counting pixels per ASIC (Llopart et al., 2001). Entire research fields would be unrecognizable without the current wide availability of photon-counting detectors: protein crystallography (Hülsen et al., 2006), industrial X-ray diffraction (de Vries et al., 2007), electron microscopy (Sikharulidze et al., 2011), medical imaging (Taguchi \& Iwanczyk, 2013), computed tomography (Pöllmann et al., 2010) and X-ray pump-probe techniques (Kenney et al., 2013).

One of the limitations of current photon-counting pixel detectors is the loss of linearity at high counting rates, followed by saturation and paralysis at even higher photon rates (Walko et al., 2008). This problem is set to increase in the near future, as many synchrotron facilities are planning substantial upgrades in brilliance (Chenevier \& Joly, 2018) and free-electron laser facilities are considering operating in a 
quasi-continuous-wave mode in the future (Brinkmann et al., 2014; Marcus \& Raubenheimer, 2017).

Ultrafast integrating pixel detectors currently under development will be able to operate at $100 \mathrm{kHz}$ and count linearly at extremely high photon fluxes towards $10^{8}$ photons per pixel per second (Blaj et al., 2014, 2015, 2016a, 2019b), while also providing full spectral information on a photon-byphoton basis (at low photon occupancies) (Blaj et al., 2016b). However, until these detectors become available and practical for applications outside the demanding area of free-electron laser applications (Graafsma, 2009; Blaj et al., 2016c), it is useful to improve the performance of currently available photon-counting (Ballabriga et al., 2016) or time-overthreshold (TOT) detectors (Llopart et al., 2007; Poikela et al., 2014; Johnson et al., 2014).

One approach to linearizing the saturation behavior of photon-counting detectors is to model the dead-time in detectors with a single counter and calculate a transformation function for linearization. Current models describing the dead-time and counting behavior of photon-counting pixel detectors are incomplete, relying on characterizing the response of a single counter in a particular detector as a function of experimental conditions, e.g. the Mythen (Bergamaschi et al., 2011), Pilatus (Trueb et al., 2012) or Eiger (Johnson et al., 2014) detectors; assuming a fixed dead-time (Bateman, 2000; Knoll, 2010; Taguchi et al., 2011); and discussing the effect of photon energy and threshold on counting with an exponential decay pulse shape for the analog signal (Bergamaschi et al., 2011). Note, however, that these detectors use an exponential decay pulse shaping (Brönnimann \& Trüb, 2014) and have only one counter (Ballabriga et al., 2016), unlike spectroscopic photon-counting detectors.

Several simplified analytical models are discussed by Walko et al. (2008): non-extended dead-time, extended dead-time and 'isolated' dead-time applicable to e.g. scintillators and avalanche photodiodes, thus progressing towards explaining the count rate of single-photon-counting detectors beyond the previous methods; however, the model only takes into account fixed extendable dead-time. The inadequacies of the current dead-time models and the limited performance in photoncounting linearization have been noted in the literature (Sobott et al., 2013).

Other approaches to linearizing the saturation behavior of photon-counting detectors include: (i) using two thresholds and summing their counting rates, which yields a modest increase in linearity (Schmitt et al., 2015); (ii) designing complex detectors combining photon-counting with TOT information (Bergamaschi et al., 2011; Schmitt et al., 2015), with performance limited by the precision of the TOT measurements (Schmitt et al., 2015); (iii) designing detectors with two different shaping times, then modeling and correcting their behavior (Abbene \& Gerardi, 2015); or (iv) using pixels with two thresholds and counters, and calculating the sum of the lower counter with a multiplication by a fixed factor with the higher counter (Kappler et al., 2010, 2012; Kraft et al., 2012), similar to (i), although it must be noted that the fixed factor is only valid at a particular photon rate.
We present here a statistical approach to modeling deadtime and counting nonlinearities for typical spectroscopic detectors (i.e. with multiple energy thresholds) and a constant current discharge of the feedback capacitor (Krummenacher, 1991; Llopart et al., 2001, 2007; Ballabriga et al., 2013) and resulting pile-up counter models. These models reproduce the characteristics of counter rates as a function of photon rate. Finally, we discuss the effect of typical synchrotron fill patterns on the counting rate. This is relevant for the entire community of photon-counting detector research, applications and their users (Ballabriga et al., 2016).

\section{Photon-counting pixel detectors}

\subsection{Analog signal}

A sensor (typically a reverse-biased $p-n$ junction) detects a photon which is converted into electron-hole pairs. Depending on the sensor, holes (usually, for $n$-type silicon sensors) or electrons drift toward the pixel flip-chip bonding pads (Blaj et al., 2017b). The charge is collected, amplified and converted to a voltage in the charge preamplifier of each pixel (Llopart et al., 2001). For simplicity, we will call the analog output voltage of the charge preamplifier the 'signal'.

Typically, the preamplifier is implemented with a constant current discharge of the feedback capacitor (Krummenacher, 1991; Llopart et al., 2001), resulting in an approximately triangular pulse shape as depicted in Fig. 1. This pulse is then compared with one or more thresholds (Ballabriga et al., 2013).

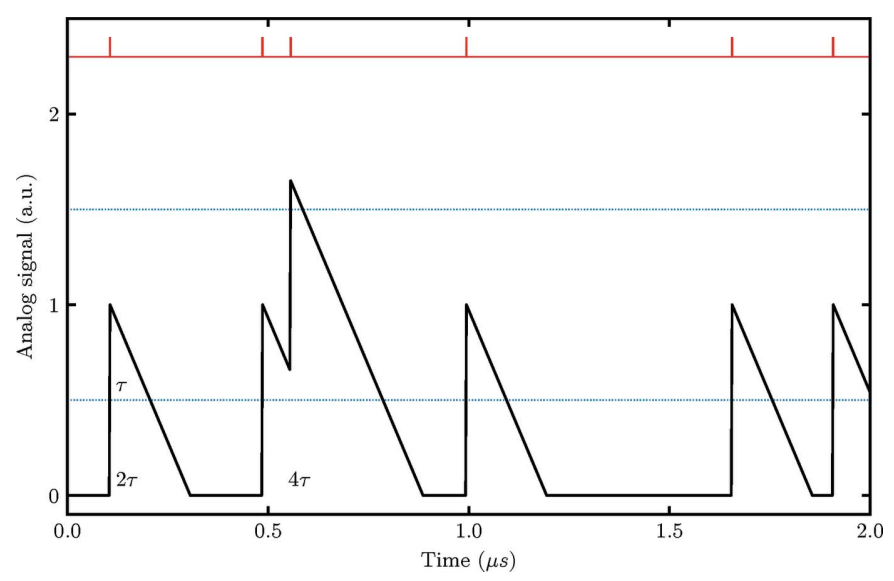

Figure 1

An example of the typical signal presented to the threshold comparator, resulting from a relatively short shaping time and a constant discharge current (black line), corresponding to discrete photon arrival times (red line). The two comparator thresholds $\left(\frac{1}{2}\right.$ and $\left.\frac{3}{2}\right)$ are indicated by the blue dotted lines. Typically, individual photons only trigger the lower threshold (most photons shown), but occasionally the time difference between two photons is short enough to lead to pile-up and triggering of the higher threshold (near $t=0.6 \mu \mathrm{s}$ ). The example shown here corresponds to a normalized photon rate $2 \lambda \tau=0.2$, where $2 \tau$ is the decay time of the analog signal from 1 to $0 . \tau$ indicates the time for the signal from one photon to return to $\frac{1}{2}, 2 \tau$ for the one-photon signal to return to 0 and $4 \tau$ for the two-photon signal to return to zero. 


\subsection{Photon-counting}

One or more threshold comparators are connected to the corresponding counters, and when the signal exceeds one of the thresholds the corresponding counter is incremented by 1 (Llopart et al., 2001). The 'photon-counting' approach thus discards small signals and the corresponding noise (along with detailed spectral information of individual photons), providing a compact lossy summary of the data (Tlustos, 2005; Blaj et al., 2016b; Michalowska-Forsyth, 2018); most of the noise is still present (superimposed on the photon signals), leading to the relatively limited spectroscopic resolution of photon-counting detectors (Tlustos, 2005).

The thresholds must be set judiciously to minimize the effect of charge sharing along the edges between two pixels; this corresponds to a setting of $\frac{1}{2}$ of the expected photon signal amplitude for detectors with a single threshold, or settings of $\frac{1}{2}$ and $\frac{3}{2}$ of the expected photon signal amplitude for detectors with two thresholds (see the blue dotted lines in Fig. 1). When each of these thresholds is exceeded, the corresponding counters $C_{0}$ and $C_{1}$ are incremented by 1 . Note that when set at the default value of $\frac{3}{2}, C_{2}$ can only be triggered by pile-up, i.e. two photons arriving within a short time $\tau$.

In most photon-counting pixel detectors this results in reduced quantum efficiency at the corners of four pixels (Gimenez et al., 2011), unless a complex 'charge-summing' mode is implemented (Ballabriga et al., 2007, 2013; Gimenez et al., 2011). More than two thresholds are available in some detectors, e.g. up to eight for the Medipix3RX (Ballabriga et al., 2013); see the review by Ballabriga et al. (2016).

\subsection{Photon pile-up}

With typical X-ray sources of constant intensity, photon detection is a Poisson process; the time intervals between successive photon arrival times $t$ follow an exponential distribution $\sim \lambda \exp (-\lambda t)$, where $\lambda$ is the average photon rate per pixel.

Single photon counts are typically obtained as $C_{0}-C_{1}$ (i.e. by subtracting photon pile-up counts $C_{1}$ from the total number of detected events $C_{0}$ ). Note that multiple comparators threshold the same signal, thus the noise corresponding to the counting statistics is limited to $1 /\left(C_{0}-C_{1}\right)^{1 / 2}$. Photon-counting detectors with a single threshold and counter could perform a two-threshold measurement sequentially, but $C_{0}$ and $C_{1}$ would be statistically independent, leading to larger errors [of the order of $\left.\left(C_{0}+C_{1}\right)^{1 / 2} /\left(C_{0}-C_{1}\right)\right]$.

Photon pile-up can either result in peak pile-up (when photons arrive at very short intervals), triggering comparators corresponding to the sum of their energies; or in spectral distortion when a second photon arrives on the tail of the pulse of a previous photon (Ballabriga et al., 2016). In either case, the pile-up can be modeled, resulting in methods to linearize the response of the counters.

In applications with a complex spectrum, the photon pile-up can be difficult to recover (Blaj et al., 2017a), especially with the lossy information collected by photon-counting detectors. Spectroscopic detectors with two or more thresholds and counters could be used to recover some of the lost information. However, many of the applications with the highest dynamic ranges use (quasi-)monochromatic radiation, e.g. protein crystallography (Hülsen et al., 2006), X-ray reflectometry (de Vries et al., 2007), X-ray computed tomography (Pöllmann et al., 2010), X-ray imaging (Procz et al., 2011) and wavelength-dispersive spectrometry (Blaj et al., 2019a), which simplifies considerably the task of estimating the effect of photon pile-up on the counters and linearizing the pixel response.

\subsection{Simulation of pile-up and effects on counters}

To test the analytical correction methods in Section 3, we implemented a Monte Carlo simulator for estimating and modeling the effect of pile-up on the counters of photoncounting pixel detectors, using several assumptions: (i) monochromatic radiation, (ii) a constant discharge current of the feedback capacitor, resulting in a triangular pulse shape with abrupt onset; (iii) a fast shaping time compared with the discharge time, resulting in a triangular pulse shape; (iv) two thresholds set at $\frac{1}{2}$ and $\frac{3}{2}$ of the pulse height for the photons, corresponding to counters $C_{0}$ and $C_{1}$, respectively; (v) the entire charge from one photon being deposited in a single pixel, thus ignoring charge sharing.

We simulated frame shutter times of $10 \mathrm{~s}$ (for improved statistics), with a time step of $10 \mathrm{~ns}$ and a dead-time of $100 \mathrm{~ns}$ (i.e. the time $\tau$ for the signal to return from 1 to $\frac{1}{2}$ and re-enable counting in $C_{0}$, or half of the time $2 \tau$ for the signal to return to the baseline). Note that the actual dead-time $\tau$ has an approximately linear dependence on photon energy. We simulated a large number of photon rates $\lambda$, varying from $5 \times 10^{3}$ to $5 \times 10^{6}$ photons per pixel per second. The results are shown in Fig. 2, with the saturation effect clearly visible

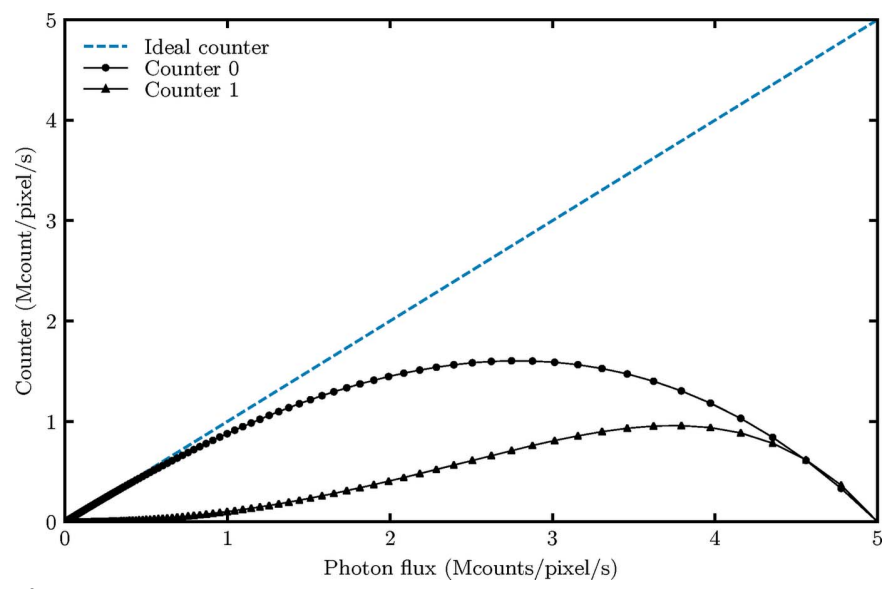

Figure 2

The simulated counter rate as a function of photon flux with $T=10 \mathrm{~s}$ and $\tau=100 \mathrm{~ns}$. Both counters $C_{0}$ and $C_{1}$ (corresponding to thresholds of $\frac{1}{2}$ and $\frac{3}{2}$, respectively) have a complex dependency on the actual photon rate $\lambda$ and deviate considerably from the ideal response (blue dashed line). When $2 \lambda \tau$ exceeds 1 (corresponding to $5 \mathrm{M}$ counts per pixel per second), the circuit is driven into saturation, resulting in counting paralysis. Note that using a single counter results in two different photon fluxes corresponding to a single counter value; using a second counter solves the confusion and effectively doubles the useful range. 
for both counters. Note the similarities and differences in the shape of counter $C_{0}$ with the experimental measurements in Fig. 6 of the paper by Sobott et al. (2013) and in Fig. 5 of the paper by Kraft et al. (2012). The similarities are due to photon pile-up, while the differences are due to the constant current discharge in Fig. 2 compared with the exponential discharge.

Note that using a single counter results in two different photon fluxes corresponding to a single counter value; using a second counter solves the confusion and effectively doubles the useful range.

While some detectors might use two thresholds to increment a single counter using counter logic (e.g. Llopart et al., 2001), their behavior is complex and beyond the scope of this article. These detectors can typically be turned into singlethreshold counters by disabling the higher threshold (Tlustos, 2005).

\subsection{Threshold equalization and gain calibration}

Variations in individual pixels result in a range of different offsets of the signal 'zero' (Ballabriga et al., 2016), with the offsets typically following a Gaussian distribution. The spread can be comparable with the photon energies to be measured. As it cannot be corrected in post-processing (like in chargeintegrating detectors) and it would severely limit the counting performance, the typical practical solution is to include a number of 'trimming bits' in each pixel to align the response of individual pixels in the matrix (Llopart et al., 2001). Often this calibration is performed with the noise edge, resulting in an equalization where the pixels end up with e.g. the $5 \sigma$ point in the noise distribution aligned; while this is not optimal, it is easy to perform.

Another effect is pixel gain dispersion (Ballabriga et al., 2016) (typically within a few percent). While its effects are limited at low energies (especially with charge-summing detectors that minimize the impact of charge sharing), at higher energies and with smaller pixels the effects become more pronounced. In imaging applications, this is often calibrated and corrected by flat-field measurements (mixing the actual flat field with the pixel gain and charge sharing).

For the individual energies of monochromatic beams, the threshold equalization can be adapted to mitigate both pixel offset and gain simultaneously. Ideally, the user should only provide the beam energy, and the corresponding equalization mask would be calculated using previously calibrated dark and gain maps. This could be achieved by carefully calibrating the pixel thresholds with different monochromatic energies or element absorption edges (Procz et al., 2009; Kraft et al., 2009) covering the entire applicable spectrum, and calculating the optimal pixel trim mask from the expected beam energy.

Alternatively, a photon-counting detector with both hardware offset and gain equalization has been developed recently (Grybos et al., 2016).

\subsection{Glossary}

Throughout this paper, we will use the following notation: $N$ : Number of photons. ${ }^{1}$

$E_{0}$ : Photon energy.

$0, \frac{1}{2}, 1, \frac{3}{2}$ : Analog signal (normalized to photons).

$C_{0}$ : Counter 0 (with signal threshold at $\frac{1}{2}$ photon).

$C_{1}$ : Counter 1 (with signal threshold at $\frac{3}{2}$ photon).

$r$ : Counter ratio $C_{1} / C_{0}$.

$\tau$ : Dead-time (proportional to photon energy $E_{0}$ ).

$2 \tau$ : Time for signal to return from 1 to baseline $(0)$.

$\lambda$ : Photon rate (photons per pixel per second).

$\Lambda$ : Photon rate (photons per pixel per pulse).

$2 \lambda \tau$ : Normalized photon rate $\in[0,1] .^{2}$

$T$ : Frame shutter time.

$g$ : Relative pixel gain (typically close to 1 ).

$\delta t$ : Gap between two buckets in a regular fill pattern.

\section{Dead-time models and counting linearization}

In the ideal case, we use monochromatic radiation with photon energy $E_{0}$ and all pixels have the same gain. For simplicity, we assume that the photon energy $E_{0}$ corresponds to an analog signal of 1 and the analog signal decay time from 1 to 0 is $2 \tau$. The detection rate is $\lambda$ photons per pixel per second and the integration time is $T$ seconds. The expected number of photons per pixel is $N=\lambda T$ (i.e. the weighted average of the corresponding Poisson distribution).

For low photon rates compared with the dead-time, $\lambda \ll 1 / \tau$, the individual photon signals will be collected separately; with the usual thresholds at $\frac{1}{2}$ and $\frac{3}{2}$, counter $C_{0}$ will contain the number of independently detected photons and counter $C_{1}$ will contain the number of occasional pile-up events when a photon arrives within time $\tau$ of another.

Counter $C_{0}$ is incremented each time the signal passes threshold 0 of $\frac{1}{2}$. This requires a starting signal under the threshold with at least one photon driving the signal over the $\frac{1}{2}$ threshold.

Counter $C_{1}$ is similarly incremented when the signal exceeds the threshold $\frac{3}{2}$, i.e. the signal is between $\frac{1}{2}$ and $\frac{3}{2}$ and one or more photons drive the signal over the $\frac{3}{2}$ threshold.

We did not implement a signal saturation limit in this simulation, as: (i) it would depend on the detector and gain mode and (ii) it would have limited effects at low photon energies (e.g. $8 \mathrm{keV})$. However, signal saturation enforces an upper limit on the random-walk effect shown in Fig. 3, resulting in more frequent returns to the baseline and effectively increasing the accuracy of the models presented here.

\subsection{Typical approach}

In the typical approach (Walko et al., 2008), a fixed paralyzable time $\tau$ is assumed. After a photon is detected, a second

\footnotetext{
${ }^{1}$ For simplicity, we use the same notation $N$ for the number of photons, regardless of whether it refers to the number of simulated photons, detected photons, expected number or linearized response; the particular meaning in each equation should be clear from the context.

${ }^{2}$ While the normalized photon rate can exceed 1 , it leads to saturation and complete counter paralysis.
} 


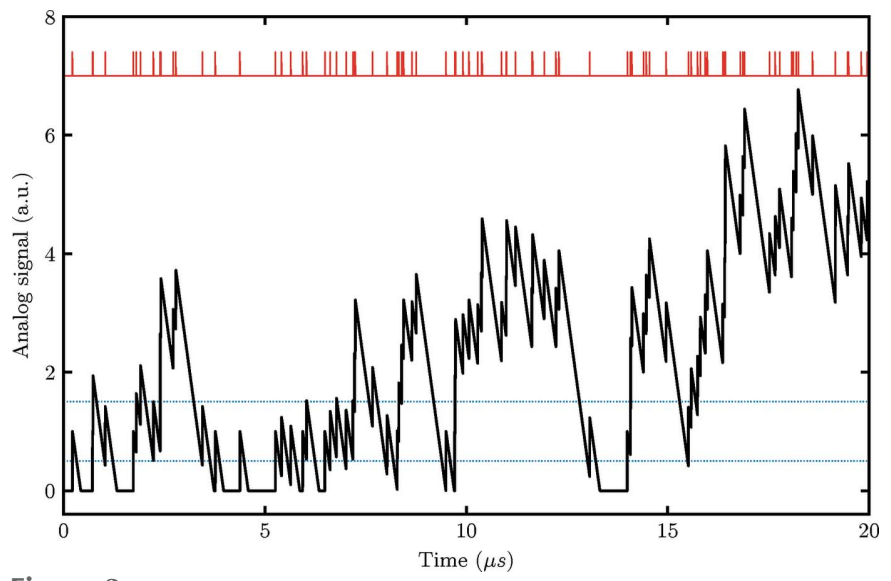

Figure 3

An example of significant pile-up corresponding to a photon rate of $2 \lambda \tau=$ 1 , where $2 \tau$ is the signal decay time of the analog signal from 1 to 0 . Note the resemblance to a random walk, resulting in an apparent baseline shift and the complete breakdown of counting over large stretches of time.

photon can arrive within the paralyzable time, leading to pileup

$$
C_{1}=\lambda T \int_{0}^{\tau} \lambda \exp (-\lambda t) \mathrm{d} t=\lambda T[1-\exp (-\lambda \tau)],
$$

or can arrive after a fixed time $\tau$, yielding

$$
C_{0}=\lambda T \int_{\tau}^{\infty} \lambda \exp (-\lambda t) \mathrm{d} t=\lambda T \exp (-\lambda \tau)
$$

resulting in a relatively simple correction formula (Schmitt et al., 2015):

$$
N=\lambda T=C_{0}+C_{1} .
$$

In Fig. 4 we compare the equations with the actual counter behaviors. Note that both equations (1) and (2) are oversimplifications, failing to match the behaviors of $C_{0}$ and $C_{1}$ for higher photon rates $\left(2 \lambda \tau\right.$ approaching $\left.\frac{1}{2}\right)$.

\subsection{Novel simple model}

Assuming a photon arriving at $t=0$ (and ignoring the effect of previously arrived photons), the signal will be higher than $\frac{1}{2}$ for $t \in[0, \tau)$ and lower than $\frac{1}{2}$ for $t \in(\tau, \infty)$. Consequently, counter $C_{0}$ can be incremented when a second photon arrives and the signal is smaller than $\frac{1}{2}$, i.e. at a time $t>\tau$ :

$$
C_{0}=\lambda T \int_{\tau}^{\infty} \lambda \exp (-\lambda t) \mathrm{d} t=\lambda T \exp (-\lambda \tau) .
$$

Incrementing $C_{1}$ requires exceeding threshold $\frac{3}{2}$ which requires at least a third photon arriving within a time $\tau$ of the second photon:

$$
C_{1}=C_{0} \int_{0}^{\tau} \lambda \exp (-\lambda t) \mathrm{d} t=\lambda T \exp (-\lambda \tau)[1-\exp (-\lambda \tau)] .
$$

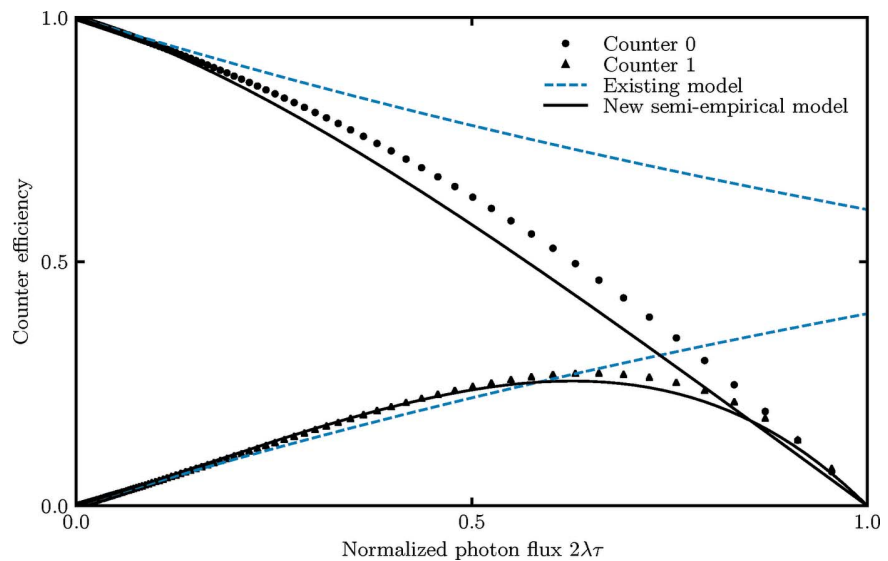

Figure 4

The counter efficiency (i.e. the ratio between counting rates and photon rates) as a function of the normalized photon rate $2 \lambda \tau$; an ideal counter would have an efficiency of 1 over the entire range. The usual models [equations (1) and (2)], depicted with dashed lines, fail to describe the counter behavior of the two counters at higher photon rates. An improved approximation [equations (19) and (20)], indicated by the solid black lines, yields much better results and forms the basis of the semiempirical model described in Section 3.4. Systematic deviations are expected, due to the apparent baseline shift at high photon rates.

$$
r=\frac{C_{1}}{C_{0}}=1-\exp (-\lambda \tau)
$$

with the corresponding number of photons

$$
N=\lambda T=\frac{C_{0}}{\exp (-\lambda \tau)}=\frac{C_{0}^{2}}{C_{0}-C_{1}}=C_{0} \frac{1}{1-r} .
$$

This works directly with pixels equalized at energy $E_{0}$. In the case of a different equalization (e.g. with noise or at a different energy), the (relatively small) variation in gain might play a role, which is discussed in Section 3.3. In this formulation, the parameters $\tau$ and $T$ drop gracefully out of the equations.

\subsection{Novel simple model with relative pixel gain}

Typically, the thresholds are set globally for each ASIC and used separately by the comparators in each pixel; the ideal values for threshold 0 (corresponding to counter $C_{0}$ ) and threshold 1 (corresponding to counter $C_{1}$ ) are $\frac{1}{2}$ and $\frac{3}{2}$, respectively (see discussion in Section 2.2). With or without an optimal threshold equalization (see Section 2.5), small residual differences in relative pixel gain could be present and these can be calibrated with a 'gain map' (Blaj et al., 2019c). Similarly, a small variance in the discharge current across pixels might be present (Ballabriga, personal communication); the effects of this variation in discharge slope are mathematically equivalent to an additional small variation in the pixel gain map.

In this subsection, we investigate the effects of gain variation on the simple model [equation (7)].

For individual pixel gain $g$ (with $g \rightarrow 1$, typically within a few percent), the equations above become

$$
C_{0}=\lambda T \int_{(g-1 / 2) 2 \tau}^{\infty} \lambda \exp (-\lambda t) \mathrm{d} t=\lambda T \exp \left[-\left(g-\frac{1}{2}\right) 2 \lambda \tau\right]
$$

The ratio of the two is 
and

$$
\begin{aligned}
C_{1} & =C_{0} \int_{0}^{(2 g-3 / 2) 2 \tau} \lambda \exp (-\lambda t) \mathrm{d} t \\
& =C_{0}\left\{1-\exp \left[-\left(2 g-\frac{3}{2}\right) 2 \lambda \tau\right]\right\} .
\end{aligned}
$$

The ratio between the two is

$$
r=\frac{C_{1}}{C_{0}}=1-\exp [-(4 g-3) \lambda \tau]
$$

resulting in

$$
\exp [-(4 g-3) \lambda \tau]=1-r
$$

By taking the natural logarithm and multiplying by $(2 g-1)$ / $(4 g-3)$ we obtain

$$
-(2 g-1) \lambda \tau=\frac{2 g-1}{4 g-3} \ln (1-r),
$$

which can be rewritten as

$$
\exp [-(2 g-1) \lambda \tau]=(1-r)^{(2 g-1) /(4 g-3)} .
$$

Finally, substituting equation (13) into equation (8) yields the linearization,

$$
N=\frac{C_{0}}{(1-r)^{(2 g-1) /(4 g-3)}},
$$

which is the general form of equation (7). Note that equation (14) cannot be used directly for e.g. three-photon pile-up; equation (9) would have to be rewritten for the pile-up of three photons.

The relative gain $g$ has a relatively small influence on the counting rate correction,

$$
\lim _{g \rightarrow 1} N=\frac{C_{0}}{1-r}[1+2(g-1) \ln (1-r)],
$$

obtained by Taylor expansion of equation (14) and keeping the first two terms; the second term is relatively small compared with 1 because $\lim _{g \rightarrow 1}(g-1)$ is small.

\subsection{Novel semi-empirical model}

The 'simple' model [equation (7)] performs well at low photon rates $2 \lambda \tau \simeq \frac{1}{2}$, but it ignores the stochastic 'baseline shift' (similar to a random walk) shown in Fig. 3. We introduce here a simplified approach to deal with this effect.

Due to the triangular pulse shape and linear behavior of the signal, the fraction of time spent above the noise floor is $2 \lambda \tau$, thus the fraction of time spent in the noise floor is:

$$
F_{0}(2 \lambda \tau)=1-2 \lambda \tau
$$

Pulses corresponding to exactly $n$ photons have a total duration of $2 n \tau$. The probability of detecting exactly $n$ photons in that time interval can be calculated by taking into account the Poisson distribution of $n$ photons in $2 n \tau$ time, modulated by the probability of being in the 'ground state' $(1-2 \lambda \tau)$, resulting in a fraction:

$$
F_{n}(2 \lambda \tau)=(1-2 \lambda \tau) \frac{(2 n \lambda \tau)^{n} \exp (-2 n \lambda \tau)}{n !}
$$

Consequently, the probability of the signal being under $\frac{1}{2}$ is approximately

$$
\begin{aligned}
p\left(s<\frac{1}{2}\right) & =F_{0}(2 \lambda \tau)+\frac{F_{1}(2 \lambda \tau)}{2}+\ldots \\
& \simeq(1-2 \lambda \tau)[1+\lambda \tau \exp (-2 \lambda \tau)]
\end{aligned}
$$

(keeping only the first two terms for simplicity). The factor 2 in the denominator corresponds to the fraction of time with signal $<\frac{1}{2}$, i.e. $\tau$ during a pulse duration $2 \tau$; using additional terms improves the model accuracy (not shown).

Multiplying the probability of a signal $s<\frac{1}{2}$ with the probability of detecting a photon while the signal is smaller than $\frac{1}{2}$,

$$
C_{0} \simeq \lambda T(1-2 \lambda \tau)[1+\lambda \tau \exp (-2 \lambda \tau)] .
$$

Incrementing $C_{1}$ requires detecting a second photon within a time $2 \tau$ of the first photon, with an empirical model

$$
C_{1} \simeq C_{0} \lambda \tau \exp (2 \lambda \tau)
$$

Note in Fig. 4 that these models (depicted by the solid lines) describe the observed behavior of Counters 0 and 1 more accurately than existing models.

The ratio of the two counters is thus

$$
r=\frac{C_{1}}{C_{0}}=\lambda \tau \exp (2 \lambda \tau) .
$$

Solving equation (21) analytically for $\lambda \tau$ yields a solution $\lambda \tau=$ $W_{0}(2 r) / 2$, with $W_{0}$ the principal (zeroth) solution of the Lambert $W$ function. However, while this model is more accurate than previous models (see Fig. 4), it is based on two simplifications: equation (18) keeps only the first term in an infinite series, and equation (20) implicitly ignores contributions of pile-up of three or more photons. These simplifications result in a somewhat inaccurate estimate of $\lambda \tau$. Using an empirical correction factor of 0.91 (obtained by least-squares fitting of the model with simulations) results in

$$
\lambda \tau \simeq 0.91 \frac{W_{0}(2 r)}{2} \text {. }
$$

Finally, rearranging the factors in equation (19) yields the linearization equation,

$$
N=\frac{C_{0}}{(1-2 \lambda \tau)[1+\lambda \tau \exp (-2 \lambda \tau)]},
$$

where $\lambda \tau$ can be substituted with the value calculated from equation (22).

\subsection{Novel empirical model}

Note that the ratio $r=C_{1} / C_{0}$ increases monotonically with photon flux (Fig. 5). This behavior can be modeled with an empirical function,

$$
\lambda \tau=f(r)=\exp \left[\sum_{i=0}^{3} a_{i}(\ln r)^{i}\right]
$$

see the fit in Fig. 5 and the resulting parameters in Table 1. We can also model the ratio $C_{0} / N$ as a function of $\lambda \tau$, 
Table 1

Fitting parameters for $f(r)$.

\begin{tabular}{lrl}
\hline Parameter & \multicolumn{1}{c}{ Value } & $\sigma$ \\
\hline$a_{0}$ & -0.7908 & 0.0008 \\
$a_{1}$ & 0.5500 & 0.0016 \\
$a_{2}$ & -0.0822 & 0.0008 \\
$a_{3}$ & -0.0050 & 0.0001 \\
\hline
\end{tabular}

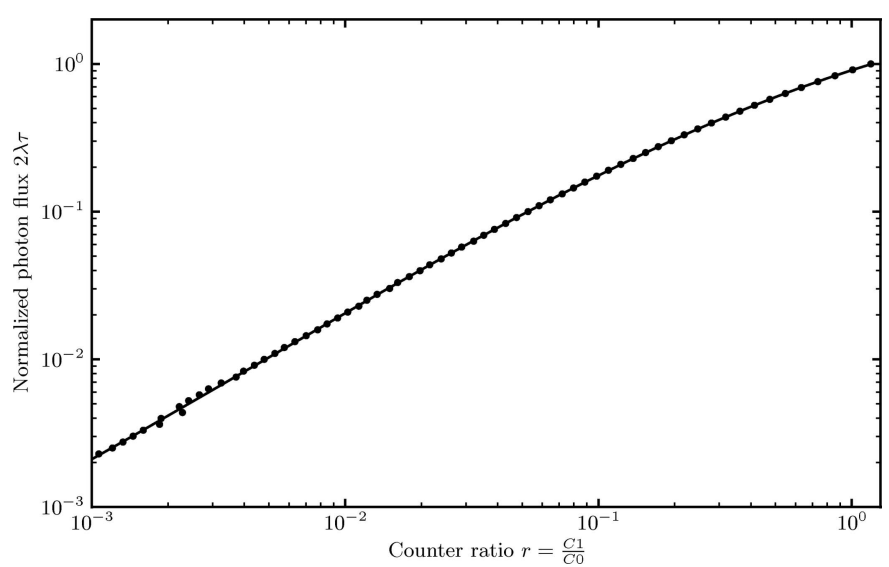

Figure 5

An empirical fit of the normalized photon flux $2 \lambda \tau$ as a function of counter ratio $r$. Black dots indicate the results of the Monte Carlo simulation and the black line depicts the value of the fitting function $f(r)$.

$$
\frac{C_{0}}{N}=g(\lambda \tau)=\sum_{i=1}^{4} b_{i}(1-2 \lambda \tau)^{i},
$$

where $b_{4}=1-b_{1}-b_{2}-b_{3}$; see the fit in Fig. 6 and the resulting parameters in Table 2 . From the ratio $r$ we can thus obtain the linearized counting rate,

$$
N=\frac{C_{0}}{g[f(r)]} .
$$

Knowledge or calibration of $\tau, T$ and $\lambda$ is not required.

3.6. Novel model for synchrotron fill patterns with widely spaced buckets

With widely spaced buckets, photons from one bucket arrive quasi-simultaneously, greatly simplifying the modeling of the counter behavior. Assuming a rate of $\Lambda$ photons per pixel per pulse and long acquisition times with relatively sparse photons in each pulse, we obtain from the Poisson distribution

$$
C_{0}=\sum_{i=1}^{\infty} \frac{\Lambda^{i} \exp (-\Lambda)}{i !}=N[1-\exp (-\Lambda)]
$$

and

$$
C_{1}=\sum_{i=2}^{\infty} \frac{\Lambda^{i} \exp (-\Lambda)}{i !}=N[1-(1+\Lambda) \exp (-\Lambda)]
$$

with ratio

$$
1-r=1-\frac{C_{1}}{C_{0}}=\frac{\Lambda \exp (-\Lambda)}{1-\exp (-\Lambda)}
$$

Table 2

Fitting parameters for $g(\lambda \tau)$.

\begin{tabular}{lcc}
\hline Parameter & Value & $\sigma$ \\
\hline$b_{1}$ & 1.584 & 0.001 \\
$b_{2}$ & -0.682 & 0.003 \\
$b_{3}$ & 0.088 & 0.005 \\
$b_{4}$ & $1-b_{1}-b_{2}-b_{3}$ & 0.006 \\
\hline
\end{tabular}

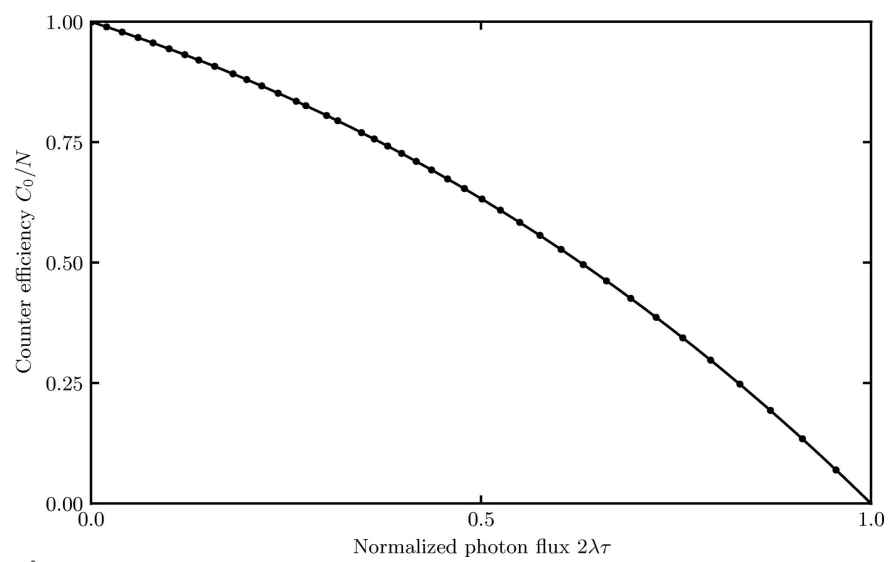

Figure 6

An empirical fit of the counter $C_{0}$ efficiency as a function of the normalized photon flux $2 \lambda \tau$. Black dots indicate the numerical results of the Monte Carlo simulation and the black line depicts the value of the fitting function $g(\lambda \tau)$.

and solution

$$
\Lambda=(r-1)-W_{-1}[(r-1) \exp (r-1)],
$$

where $W_{-1}$ is the -1 th solution of the Lambert $W$ function. This solution can be used to correct the number of pulses in $C_{0}$,

$$
\begin{aligned}
N & =\frac{C_{0}}{1-\exp (-\Lambda)} \\
& =\frac{C_{0}}{1-\left((r-1) /\left\{W_{-1}[(r-1) \exp (r-1)]\right\}\right)} .
\end{aligned}
$$

Note that this result is applicable for light sources with a (relatively) constant intensity of individual pulses. This is not typically true at free-electron laser sources, where either a more complex gamma distribution should be used instead of the Poisson distribution (Blaj et al., 2017a) or individual pulses should be read out separately.

\section{Results}

In Fig. 7 we show the typical response of counter $C_{0}$ along with the effect of the various counting-rate corrections, including the counting statistics noise, which is obtained by simulating 100 acquisitions at each point, applying corrections to each result, and using the mean and the standard deviation of each set to estimate the systematic deviations (markers) and the counting noise (error bars), respectively. The ideal response is indicated by the thick solid blue line at $y=1$; models closer to the ideal perform better (i.e. their systematic deviation is smaller). 


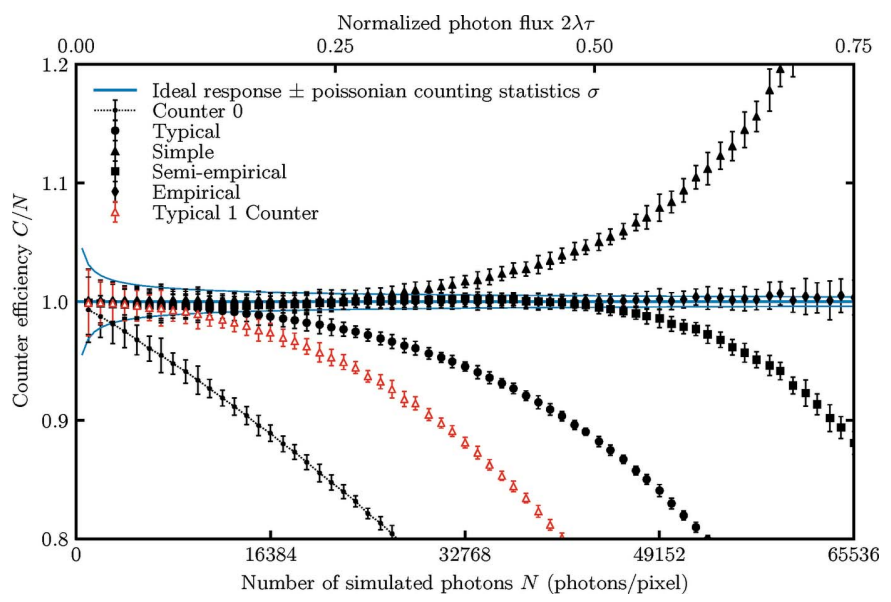

Figure 7

A comparison of the counting efficiencies with and without corrections for realistic parameters $(\tau=100 \mathrm{~ns}$, integration time $T=20 \mathrm{~ms}, \lambda \in[0$, $3.25 \mathrm{M}$ counts per pixel per second], pixel counter depth 65536). The ideal counter efficiency is 1 over the whole range (thick solid blue line), with the counting statistics limits $\pm \sigma$ indicated by thin solid blue lines. Error bars represent the counting statistics errors after linearization. The uncorrected counter quickly deviates from the ideal (small circles with dotted line). A corrected single counter (open red triangles) requires knowledge of the parameters $\tau$ and $T$ and underperforms a simple sum of the two counters (large circles). Various two-counter dead-time corrections yield increasingly accurate results, extending the linear counting rate by one to two orders of magnitude (empirical model, black diamonds) and enabling linear counting rates in excess of $10^{7}$ counts per pixel per second.

\subsection{One counter}

Linearizing the response of single photon-counting pixel detectors with a single counter requires knowledge of the integration time $T$ and dead-time $2 \tau$, and can only be used for $\lambda \ll 1 / 4 \tau$. This could be achieved by solving equation (4) using the (assumed known) $T$ and $\tau$ parameters, resulting in an estimated number of photons,

$$
N=-\frac{T}{\tau} W_{0}\left(-\frac{C_{0} \tau}{T}\right) .
$$

In practice, an empirical calibration is likely to yield superior results, due to the mismatch between the model in equation (4) and the actual behavior of $C_{0}$. The calibration would depend on the parameters $\tau$ and $T$, as well as on the fill pattern of the light source; see the discussion in Section 4.3.

\subsection{Two counters}

Using two counters eliminates the need to know or calibrate $E_{0}$ (the photon energy) ${ }^{\mathbf{3}}, 2 \tau\left(E_{0}\right)$ (the time for the analog signal to return from 1 to 0 ) and $T$ (the integration time). More accurate models perform well at rates approaching $2 \lambda \tau \rightarrow \frac{1}{2}$. Using any linearization approach yields superior results compared with no linearization. Even the simple sum of two counters exceeds the performance of the analytical one-

\footnotetext{
${ }^{3}$ Note, however, that the comparator thresholds for $C_{0}$ and $C_{1}$ should always be set to $\frac{1}{2}$ and $\frac{3}{2}$ of the signal, corresponding to one photon of energy $E_{0}$, as discussed in Section 2.2.
}

Table 3

Performance of photon-counting linearization methods.

$r=C_{1} / C_{0}$ and $x=0.91 W_{0}(2 r) / 2$, with $W$ the Lambert $W$ function.

\begin{tabular}{lllr}
\hline Model & Equation & Section & $\begin{array}{r}\text { Linear } \\
\text { range }\end{array}$ \\
\hline None, one counter & $C_{0}$ & 2.3 & $3.5 \%$ \\
Typical, one counter & $N\left(C_{0}, \tau, T\right)$ & 4.1 & $11.0 \%$ \\
Typical & $C_{0}+C_{1}$ & 3.1 & $15.1 \%$ \\
Novel simple & $C_{0} /(1-r)$ & 3.2 & $29.7 \%$ \\
Novel simple gain & $C_{0} /(1-r)^{(2 g-3 / 2) /(g-1 / 2)}$ & 3.3 & $29.7 \%$ \\
Novel semi-empirical & $C_{0} /\{(1-2 x)[1+x \exp (-2 x)]\}$ & 3.4 & $50.0 \%$ \\
Novel empirical & $C_{0} / g(f(r))$ & 3.5 & $64.5 \%$ \\
\hline
\end{tabular}

counter model [which also requires knowledge of $E_{0}, \tau\left(E_{0}\right)$ and $T]$.

The simple model (indicated by filled triangles in Fig. 7) offers an increased linear range with minimal implementation effort and outperforms all previous models, but note the overestimation of the counting rates.

The semi-empirical and empirical models (depicted in Fig. 7 by filled squares and diamonds, respectively) offer the best performance. In particular, the empirical model exceeds by far the performance of the other models. Using the semiempirical or empirical linearization methods with e.g. the Medipix3RX with a maximum (i.e. nonlinear) counting rate of $4.5 \times 10^{6}$ counts per pixel per second (Ballabriga et al., 2016) results in a linear counting rate in excess of $10^{7}$ counts per pixel per second.

In Table 3, the performance of each linearization model is summarized; the linear range is defined as the range where systematic deviations are limited to the photon-counting statistics (indicated by the thin solid blue lines in Fig. 7). The empirical model yields an improvement of one to two orders of magnitude in the linear range compared with a single counter, with an error comparable with the counting statistics for normalized photon rates up to $2 \lambda \tau \simeq 0.6$.

\subsection{Synchrotron fill patterns}

Synchrotron fill patterns influence the behavior of the counters. For regular fill patterns and closely spaced buckets, e.g. typical gaps $\delta t \simeq 2 \mathrm{~ns}$ at synchrotrons, much smaller than the dead-times $\tau \simeq 100 \mathrm{~ns}(\delta t \ll \tau)$, the equations above remain valid.

With larger gaps approaching $\tau(\delta t<\tau / 2)$, the equations describing the behavior of individual counters have to be corrected. Let $f$ be the fraction of time with beam and $1-f$ the fraction of time with gaps; then we can substitute $\lambda$ with $\lambda / f$ and $T$ with $f T$. Note that in detectors with two thresholds $\tau, T$ and $f$ drop out of the linearization equations (3), (7), (14), (23) and (26), leading to exactly the same result.

For large intervals between buckets, $\delta t \gg \tau$, the dead-time is irrelevant and the pile-up is reduced to the elegant solution in equation (31).

Finally, for other fill patterns $(e . g . \delta t \simeq \tau)$, the Poisson distribution (or gamma distribution in the case of free-electron laser beams) in each bucket needs to be estimated. Alternatively, the correspondence between the ratio $C_{1} / C_{0}$ 
and the ratio $N / C_{0}$ can be calibrated empirically as a function of beam intensity, as shown in Section 3.5, yielding a calibration curve specific to the actual fill pattern.

\subsection{Charge sharing}

Charge sharing is a complex phenomenon, with single photons resulting in charge clouds with sizes depending on the photon energy $\left(E_{0}\right)$, the photon interaction depth in the sensor, the sensor thickness and resistivity, the bias voltage, the pixel size etc. (Blaj et al., 2017b). Currently there is no simple method to estimate the distribution of charge cloud sizes as a function of all the parameters above. This dependence is typically ignored in the dead-time literature, presumably due to its complexity.

We repeated the simulations above with one example of realistic charge sharing using $55 \mu \mathrm{m}$ pixels and Gaussian charge clouds with $\sigma=5 \mu \mathrm{m}$ (Blaj et al., 2017b) (results not shown). Simulating detection with or without charge sharing results in differences of up to $4 \%$ in linearized responses with normalized photon fluxes $2 \lambda \tau$ up to 0.5 , and up to $10 \%$ for higher fluxes. More systematic studies are underway. Nevertheless, applying the novel linearization corrections described here still yields results much closer to the incoming photon rates than no correction at all. Moreover, in the particular case of the Medipix3 detectors, the charge summing mode (Gimenez et al., 2011) reverses the effects of charge sharing in the analog circuitry before the threshold comparators, thus avoiding the effect of charge sharing on counter values.

\section{Conclusions}

In the last decade, a revolution in hybrid pixel detectors has led to the wide availability of photon-counting pixel detectors that have enabled rapid advances in photon science and applications of high-dynamic-range imaging.

In the near future, significant brilliance upgrades are expected for most storage-ring X-ray sources (Chenevier \& Joly, 2018). High-repetition-rate free-electron lasers might also eventually operate in a (quasi-)continuous-wave mode (Marcus \& Raubenheimer, 2017; Brinkmann et al., 2014). This will further increase the pressure to linearize the saturation response of photon-counting pixel detectors, or possibly replace them with fast integrating detectors.

One existing approach to linearize the photon-counting response is to assume a simple paralyzable model response with a fixed dead-time $\tau$; however, a typical photon-counting detector with multiple thresholds has a constant current discharge of the feedback capacitor, resulting in very different statistics. Another existing approach to linearize the photoncounting response is to combine a single counter with a timeover-threshold counter. This approach increases the counting linearity, but its range is limited by the depth of the time-overthreshold counter and the signal-to-noise performance is limited by the higher noise in time-over-threshold compared with photon-counting.

In this paper we have introduced new, more accurate, statistical descriptions of the dead-time and photon pile-up in photon-counting detectors with multiple thresholds, describing the counting behavior of typical photon-counting detectors with two independent counters (corresponding to two different thresholds). Subsequently, we have derived three nonlinearity correction equations, ranging from simple to complex, which significantly extend the region with linear photon-counting compared with previous approaches. These equations allow a doubling of maximum counting rates compared with detectors with a single counter and yield an increase in linear counting rates of one to two orders of magnitude for quasi-continuous light sources.

Additionally, in detectors with at least two thresholds and counters, these linearization methods are very robust and do not require calibration or knowledge of the individual system parameters (integration time, dead-time etc.), thus enabling simple implementation or easy reprocessing of previous experimental data.

Finally, we have described the influence of synchrotron fill patterns on the photon-counting linearization methods, showing that they can be applied in a majority of cases (closely spaced buckets, approaching typical detector dead-times of $\sim 100 \mathrm{~ns}$ ). We have developed a new model for widely spaced buckets. Finally, for bucket spacings similar to the detector dead-time, we have described an empirical calibration method.

These novel methods enable extending the range of linear counting by one to two orders of magnitude for photoncounting detectors with a constant current discharge.

\section{Acknowledgements}

SLAC publication number SLAC-PUB-17431. This work was performed in support of the LCLS and SSRL projects at SLAC. The author would like to thank R. Ballabriga and L. Tlustos at CERN for many interesting discussions on photon-counting and pixel detectors.

\section{Funding information}

This work was supported by the US Department of Energy, Office of Science, Office of Basic Energy Sciences under contract Nos. DE-AC02-76SF00515 and DE-FOA-0001794.

\section{References}

Abbene, L. \& Gerardi, G. (2015). J. Synchrotron Rad. 22, 1190-1201. Anghinolfi, F., Aspell, P., Bass, K., Beusch, W., Bosisio, L., Boutonnet, C., Burger, P., Campbell, M., Chesi, E., Claeys, C., Clemens, J. C., Cohen Solal, M., Debusschere, I., Delpierre, P., Di Bari, D., Dierickx, B., Enz, C. C., Focardi, E., Forti, F., Gally, Y., Glaser, M., Gys, T., Habrard, M. C., Heijne, E. H. M., Hermans, L., Hurst, R., Inzani, P., Jaeger, J. J., Jarron, P., Krummenacher, F., Lemeilleur, F., Lenti, V., Manzari, V., Meddeler, G., Morando, M., Munns, A., Nava, F., Navach, F., Neyer, C., Ottaviani, G., Pellegrini, F., Pengg, F., Perego, R., Pindo, M., Potheau, R., Quercigh, E., Redaelli, N., Rossi, L., Sauvage, D., Segato, G., Simone, S., Stefanini, G., Tonelli, G., Vanstraelen, G., Vegni, G., Verweij, H., Viertel, G. M. \& Waisbard, J. (1992). IEEE Trans. Nucl. Sci. 39, 654-661.

Ballabriga, R., Alozy, J., Blaj, G., Campbell, M., Fiederle, M., Frojdh, E., Heijne, E. H. M., Llopart, X., Pichotka, M., Procz, S., Tlustos, L. \& Wong, W. (2013). J. Instrum. 8, C02016. 
Ballabriga, R., Alozy, J., Campbell, M., Frojdh, E., Heijne, E., Koenig, T., Llopart, X., Marchal, J., Pennicard, D., Poikela, T., Tlustos, L., Valerio, P., Wong, W. \& Zuber, M. (2016). J. Instrum. 11, P01007.

Ballabriga, R., Campbell, M., Heijne, E., Llopart, X. \& Tlustos, L. (2007). IEEE Trans. Nucl. Sci. 54, 1824-1829.

Bateman, J. E. (2000). J. Synchrotron Rad. 7, 307-312.

Bergamaschi, A., Dinapoli, R., Greiffenberg, D., Henrich, B., Johnson, I., Mozzanica, A., Radicci, V., Schmitt, B., Shi, X. \& Stoppani, L. (2011). J. Synchrotron Rad. 18, 923-929.

Blaj, G., Bhogadi, D., Chang, C.-E., Doering, D., Kenney, C., Kroll, T., Segal, J., Sokaras, D. \& Haller, G. (2019a). AIP Conf. Proc. 2054, 060037.

Blaj, G., Caragiulo, P., Carini, G., Carron, S., Dragone, A., Freytag, D., Haller, G., Hart, P. A., Herbst, R., Herrmann, S., Hasi, J., Kenney, C. J., Markovic, B., Nishimura, K., Osier, S., Pines, J., Segal, J., Tomada, A. \& Weaver, M. (2014). Synchrotron Radiation News, 27(4), 14-19.

Blaj, G., Caragiulo, P., Carini, G., Carron, S., Dragone, A., Freytag, D., Haller, G., Hart, P., Hasi, J., Herbst, R., Herrmann, S., Kenney, C., Markovic, B., Nishimura, K., Osier, S., Pines, J., Reese, B., Segal, J., Tomada, A. \& Weaver, M. (2015). J. Synchrotron Rad. 22, 577583.

Blaj, G., Caragiulo, P., Carini, G., Dragone, A., Haller, G., Hart, P., Hasi, J., Herbst, R., Kenney, C. J., Markovic, B., Nishimura, K., Pines, J., Segal, J., Tamma, C. \& Tomada, A. (2016a). AIP Conf. Proc. 1741, 040012.

Blaj, G., Caragiulo, P., Dragone, A., Haller, G., Hasi, J., Kenney, C. J., Kwiatkowski, M., Markovic, B., Segal, J. \& Tomada, A. (2016b). Proc. SPIE, 9968, 99680J.

Blaj, G., Carini, G., Carron, S., Haller, G., Hart, P. A., Hasi, J., Herrmann, S., Kenney, C. J., Segal, J., Stan, C. A. \& Tomada, A. (2016c). IEEE Trans. Nucl. Sci. 63, 1818-1826.

Blaj, G., Dragone, A., Kenney, C., Abu-Nimeh, F., Caragiulo, P., Doering, D., Kwiatkowski, M., Markovic, B., Pines, J., Weaver, M., Boutet, S., Carini, G., Chang, C.-E., Hart, P., Hasi, J., Hayes, M., Herbst, R., Koglin, J., Nakahara, K., Segal, J. \& Haller, G. (2019b). AIP Conf. Proc. 2054, 060062.

Blaj, G. Haller, G. Kenney, C. (2019c). arXiv:1907.07125v1.

Blaj, G., Kenney, C., Dragone, A., Carini, G., Herrmann, S., Hart, P., Tomada, A., Koglin, J., Haller, G., Boutet, S. M. M., Messerschmidt, M., Williams, G., Chollet, M., Dakovski, G., Nelson, S., Pines, J., Song, S. \& Thayer, J. (2017a). IEEE Trans. Nucl. Sci. 64, 28542868.

Blaj, G., Segal, J., Kenney, C. \& Haller, G. (2017b). arXiv:1706.01429. Brinkmann, R., Schneidmiller, E., Sekutowicz, J. \& Yurkov, M. (2014). Nucl. Instrum. Methods Phys. Res. A, 768, 20-25.

Brönnimann, C. \& Trüb, P. (2014). Synchrotron Light Sources and Free-Electron Lasers: Accelerator Physics, Instrumentation and Science Applications, edited by E. Jaeschke, S. Khan, J. R. Schneider \& J. B. Hastings, pp. 1-29. Heidelberg: Springer.

Chenevier, D. \& Joly, A. (2018). Synchrotron Radiation News, 31(1), 32-35.

Gimenez, E. N., Ballabriga, R., Campbell, M., Horswell, I., Llopart, X., Marchal, J., Sawhney, K. J., Tartoni, N. \& Turecek, D. (2011). IEEE Trans. Nucl. Sci. 58, 323-332.

Graafsma, H. (2009). J. Instrum. 4, P12011.

Grybos, P., Kmon, P., Maj, P. \& Szczygiel, R. (2016). IEEE Trans. Nucl. Sci. 63, 1155-1161.

Heijne, E. H., Jarron, P., Olsen, A. \& Redaelli, N. (1988). Nucl. Instrum. Methods Phys. Res. A, 273, 615-619.

Hülsen, G., Brönnimann, C., Eikenberry, E. F. \& Wagner, A. (2006). J. Appl. Cryst. 39, 550-557.

Johnson, I., Bergamaschi, A., Billich, H., Cartier, S., Dinapoli, R., Greiffenberg, D., Guizar-Sicairos, M., Henrich, B., Jungmann, J.,
Mezza, D., Mozzanica, A., Schmitt, B., Shi, X. \& Tinti, G. (2014). J. Instrum. 9, C05032.

Kappler, S., Glasser, F., Janssen, S., Kraft, E. \& Reinwand, M. (2010). Proc. SPIE, 7622, $76221 \mathrm{Z}$.

Kappler, S., Hannemann, T., Kraft, E., Kreisler, B., Niederloehner, D., Stierstorfer, K. \& Flohr, T. (2012). Proc. SPIE, 8313, 83130X.

Kenney, C. J., Dragone, A. B., Segal, J. D., Hasi, J., Mehta, A., Reis, D. A., Markovic, B., Caraguilo, P., Carini, G., Herrmann, S. C., Lindenberg, A. M. \& Haller, G. (2013). 2013 IEEE Nuclear Science Symposium and Medical Imaging Conference (2013 NSS/MIC), pp. 1-2. IEEE.

Knoll, G. F. (2010). Radiation Detection and Measurement. New York: John Wiley \& Sons.

Kraft, E., Glasser, F., Kappler, S., Niederloehner, D. \& Villard, P. (2012). Proc. SPIE, 8313, 83134A.

Kraft, P., Bergamaschi, A., Bronnimann, C., Dinapoli, R., Eikenberry, E., Graafsma, H., Henrich, B., Johnson, I., Kobas, M., Mozzanica, A., Schleputz, C. M. \& Schmitt, B. (2009). IEEE Trans. Nucl. Sci. 56, 758-764.

Krummenacher, F. (1991). Nucl. Instrum. Methods Phys. Res. A, 305, $527-532$.

Llopart, X., Ballabriga, R., Campbell, M., Tlustos, L. \& Wong, W. (2007). Nucl. Instrum. Methods Phys. Res. A, 581, 485-494.

Llopart, X., Campbell, M., San Segundo, D., Pernigotti, E. \& Dinapoli, R. (2001). Nucl. Sci. Symp. Conf. Rec. 3, 1484-1488.

Marcus, G. \& Raubenheimer, T. (2017). ICFA Beam Dyn. Newsl. 70, 82-102.

Michalowska-Forsyth, A. (2018). Nucl. Instrum. Methods Phys. Res. A, 912, 167-173.

Poikela, T., Plosila, J., Westerlund, T., Campbell, M., Gaspari, M. D., Llopart, X., Gromov, V., Kluit, R., Beuzekom, M., Zappon, F., Zivkovic, V., Brezina, C., Desch, K., Fu, Y. \& Kruth, A. (2014). J. Instrum. 9, C05013.

Pöllmann, H., Meier, R., Blaj, G. \& Riedl, U. (2010). Adv. X-ray Anal. 54, 101-107.

Procz, S., Lubke, J., Zwerger, A., Mix, M. \& Fiederle, M. (2009). IEEE Trans. Nucl. Sci. 56, 1795-1799.

Procz, S., Pichotka, M., Lübke, J., Hamann, E., Ballabriga, R., Blaj, G., Campbell, M., Fauler, A., Mix, M., Zwerger, A. \& Fiederle, M. (2011). IEEE Trans. Nucl. Sci. 58, 3182-3189.

Schmitt, B., Bergamaschi, A., Mozzanica, A. \& Dinapoli, R. (2015). US Patent 9121955.

Sikharulidze, I., van Gastel, R., Schramm, S., Abrahams, J., Poelsema, B., Tromp, R. \& van der Molen, S. (2011). Nucl. Instrum. Methods Phys. Res. A, 633, S239-S242.

Sobott, B. A., Broennimann, Ch., Schmitt, B., Trueb, P., Schneebeli, M., Lee, V., Peake, D. J., Elbracht-Leong, S., Schubert, A., Kirby, N., Boland, M. J., Chantler, C. T., Barnea, Z. \& Rassool, R. P. (2013). J. Synchrotron Rad. 20, 347-354.

Taguchi, K. \& Iwanczyk, J. S. (2013). Med. Phys. 40, 100901.

Taguchi, K., Zhang, M., Frey, E. C., Wang, X., Iwanczyk, J. S., Nygard, E., Hartsough, N. E., Tsui, B. M. \& Barber, W. C. (2011). Med. Phys. 38, 1089-1102.

Tlustos, L. (2005). Performance and limitations of high granularity single photon processing $X$-ray imaging detectors. $\mathrm{PhD}$ thesis, Atominstitut, TU Wien, Vienna, Austria.

Trueb, P., Sobott, B. A., Schnyder, R., Loeliger, T., Schneebeli, M., Kobas, M., Rassool, R. P., Peake, D. J. \& Broennimann, C. (2012). J. Synchrotron Rad. 19, 347-351.

Vries, R. I. de, Weijers, S., Bethke, K., Kogan, V., Vasterink, J., Kharchenko, A., Fransen, M. \& Bethke, J. (2007). Nucl. Instrum. Methods Phys. Res. A, 576, 164-168.

Walko, D. A., Arms, D. A. \& Landahl, E. C. (2008). J. Synchrotron Rad. 15, 612-617. 